\title{
New Multidrug-Resistant Salmonella enterica Serovar Anatum Clone, Taiwan, 2015-2017
}

\section{Chien-Shun Chiou, Yu-Ping Hong, Ying-Shu Liao, You-Wun Wang, Yueh-Hua Tu, Bo-Han Chen, Yi-Syong Chen}

In 2011, a Salmonella enterica serovar Anatum clone emerged in Taiwan. During 2016-2017, infections increased dramatically, strongly associated with emergence and spread of multidrug-resistant strains with a plasmid carrying 11 resistance genes, including $b / a_{\mathrm{DHA}-1}$. Because these resistant strains infect humans and food animals, control measures are urgently needed.

Calmonella, a prevalent foodborne pathogen that causes $\checkmark$ zoonoses worldwide, comprises 2 species, Salmonella enterica and $S$. bongori, and $\approx 2,600$ serovars (1). In Taiwan, salmonellosis has been primarily caused by the S. enterica serovars Enteritidis, Typhimurium, Stanley, Newport, and Albany, which together caused 70\% of salmonellosis infections during 2004-2012 (2). During this period, Salmonella Anatum was not prevalent, causing only $0.4 \%$ of the infections. However, since 2015, Salmonella Anatum infections have increased, and most isolates are multidrug resistant (MDR). We report the epidemiologic trend of Salmonella Anatum infection of humans, the clonal relationships among strains recovered during 2004-2017, and the resistance mechanism of the newly emerging MDR strains.

\section{The Study}

To investigate the epidemiologic trend, we analyzed the data in the Salmonella fingerprint database constructed by the Taiwan Centers for Disease Control. The database comprises demographic and experimental data, including pulsed-field gel electrophoresis (PFGE) fingerprints obtained by using the PulseNet standardized PFGE protocol (3), serotypes obtained using PFGE pattern comparison and conventional methods (4), and antimicrobial drug susceptibility testing results for isolates collected from hospitals nationwide. We conducted whole-genome sequencing for 68 Salmonella Anatum isolates from humans and animals and 9 isolates from chicken carcasses and abbatoir environments by using the Illumina MiSeq

Author affiliation: Centers for Disease Control, Taichung, Taiwan

DOI: https://doi.org/10.3201/eid2501.181103 platform (https://www.illumina.com) and identified resistance genes, incompatibility groups of plasmids, and sequence types by using the whole-genome sequencing data. To investigate clonal relationships and locations of resistance genes, we constructed a dendrogram for Salmonella Anatum strains with whole-genome singlenucleotide polymorphism profiles to assess genetic relatedness among strains and determined the complete genomic sequence of Salmonella Anatum strain R16.0676 with whole-genome sequencing data generated by using a MinION nanopore sequencer (https://nanoporetech. com/products/minion) and an Illumina MiSeq sequencer. To investigate mobility of resistance plasmids, we conducted conjugation experiments to transfer the resistance genes-carrying (R) plasmid from Salmonella Anatum strain R16.0676 into recipient Escherichia coli C600 and transferred an R plasmid from an E. coli transconjugant back to a rifampin-resistant mutant of Salmonella Anatum strain R13.0957 (Appendix, https://wwwnc.cdc.gov/EID/ article/25/1/18-1103-App1.pdf).

The Salmonella fingerprint database of the Taiwan Centers for Disease Control contained PFGE fingerprints for 34,160 Salmonella isolates recovered during 20042017, of which antimicrobial drug sensitivity test results were available for 23,018. Salmonella Anatum was not a prevalent serovar among those collected during 20042014 (Figure 1). However, the number of Salmonella Anatum infections increased in 2015 and subsequently underwent another sharp increase in 2016 and 2017. In 2017, Salmonella Anatum accounted for $14.2 \%$ of Salmonella infections in Taiwan and ranked as the third most frequently identified serovar.

Whole-genome single-nucleotide polymorphism analysis of Salmonella Anatum recovered from humans during 2004-2017 revealed 3 distinct lineages (Figure 2). Strains of lineage (L) 1 were either pansusceptible or MDR; they mostly appeared during 2004-2009 (Appendix Table 2). L2 comprised only 2 isolates, which emerged in 2005 and were pansusceptible. L3 comprised 2 sublineages; sublineage (SL) 3 1, first detected in 2011, was mostly pansusceptible, whereas SL3 2, which first emerged in 2013, was mostly MDR. The MDR strains of SL3_2 first appeared in 2015 and were resistant or of reduced susceptibility to 10 of the 14 antimicrobial drugs tested. SMX.642 was the predominant MDR strain, but the first 2 isolates recovered 


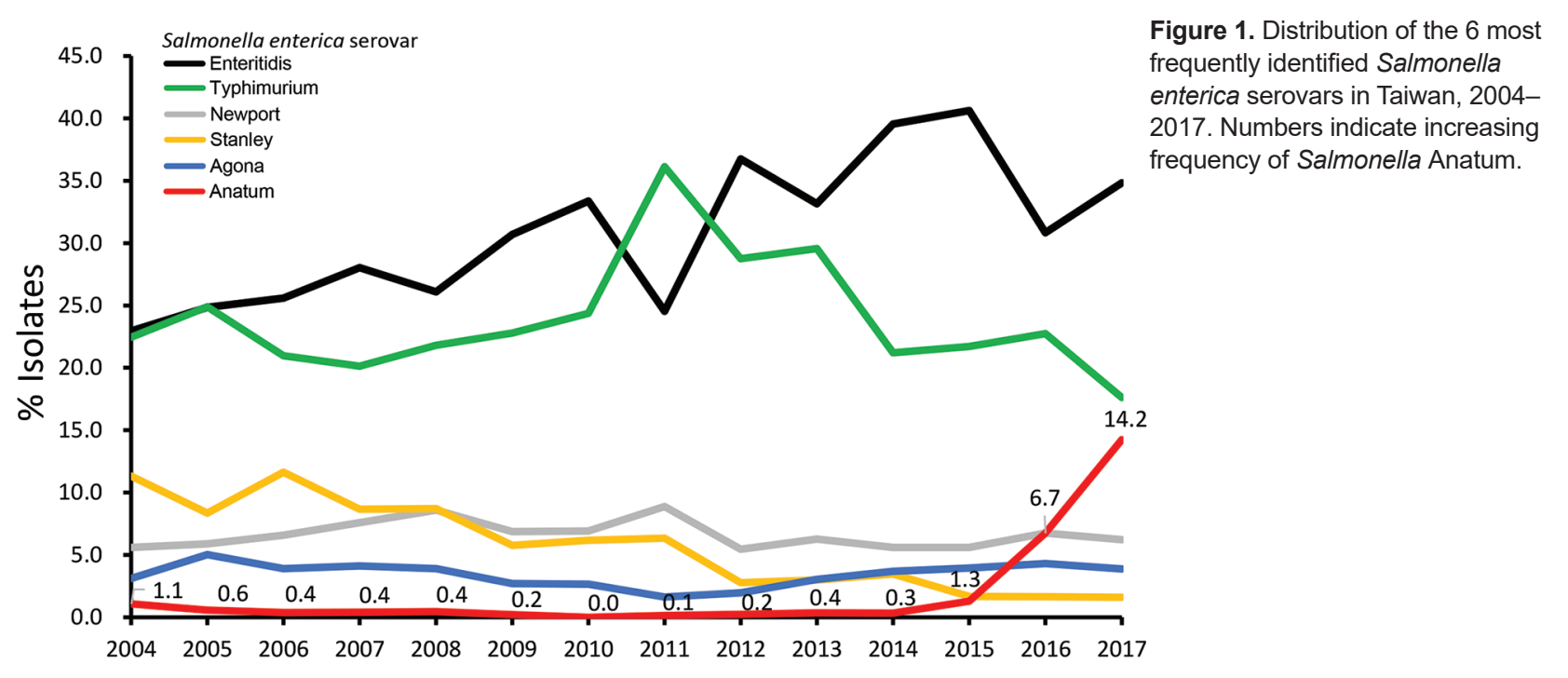

in 2013 were pansusceptible. Of the 9 isolates from chicken carcasses and abattoir environments, 5 belonged to SL3 1 and 4 to SL3 2. The new clone (L3) accounted for $91.9 \%$ of the total Salmonella Anatum infections during 2004-2017 and $99.6 \%$ in 2017. MDR strains accounted for $90.3 \%$ of the new clone recovered during 2011-2017 and $94.1 \%$ in 2017. All Salmonella Anatum isolates sequenced belonged to sequence type 64 .

The chromosomal sequence of strain R16.0676 was 4,674,190 bp (GenBank accession no. CP029800) and was not noted to carry any horizontally transferable resistance gene. R16.0676 harbored 2 plasmids, which were designated pR16.0676_90k (90,137 bp; IncC; accession no. CP029802) and $\mathrm{pR} 16.0676 \_34 \mathrm{k}(34,063 \mathrm{bp}$; IncN3; accession no. CP029801). pR16.0676 90k harbored 11 resistance genes, addA2, bla ${ }_{\mathrm{DHA}-1}, \operatorname{dfr} \bar{A} 23, \operatorname{floR}, \ln u(F)$, qnrB4, strA, strB, sull, sul2, and tet(A), which were distributed in 2 antimicrobial resistance islands, ARI1 and ARI2 (Appendix Figure, panel A). ARI1 carried 5 resistance genes, $\operatorname{floR}$, strA, strB, sul2, and $\operatorname{tet}(A)$, and was found in many IncC plasmids in the National Center for Biotechnology Information database (5). ARI2 carried the other 6 resistance genes, aadA2, bla $a_{\text {DHA-1 }}, d f r A 23, \ln u(F)$, $q n r B 4$, and sull. The resistance genes could confer resistance to cefoxitin, cefotaxime, ceftazidime, ampicillin, chloramphenicol, streptomycin, sulfonamide, tetracycline, and trimethoprim and reduced susceptibility to ciprofloxacin as shown by antimicrobial susceptibility testing (Figure 2). pR16.0676_90k shared 79\% sequence identity with a 272-kb plasmid, pECAZ155_KPC (GenBank accession no. CP019001.1), which harbored only the sequence of ARI1 but not ARI2.pR16.0676_34k did not carry any resistance gene (Appendix Figure, panel B), but it shared $98 \%$ sequence identity with a $34.8-\mathrm{kb}$ plasmid, pN-Cit (GenBank accession no. JQ996149.1).
All MDR SL3_2 isolates, including the 4 isolates recovered from the abattoirs, harbored an IncC plasmid and the same 11 resistance genes identified in strain R16.0676. Strain R17.0132 acquired an additional $\mathrm{mcr}-1$ gene and was resistant to colistin (Figure 2). We did not obtain any transconjugants with pR16.0676_90k, but we did obtain a transconjugant with a composite plasmid, which had the same sequences as pR16.0676_90k and pR16.0676_34k (Appendix Figure, panel C). This 125$\mathrm{kb}$ composite plasmid probably resulted from insertion of pR16.0676_90k into pR16.0676_34k through an insertion sequence $26-$ mediated transposition process. The resulting plasmid acquired an additional copy of insertion sequence 26 and an 8-bp tandem repeat in the insertion site. More than a dozen genes are typically required for conjugation (6). pR16.0676_90k harbored only 3 genes, and pR16.0676_34k contained at least 12 genes related to conjugation. Fusion of the 2 plasmids caused the composite plasmid to become self-transmissible. When the composite plasmid was transferred back into a rifampinresistant mutant of Salmonella Anatum strain R13.0957, we obtained transconjugants harboring only a $58-\mathrm{kb}$ or 83-kb R plasmid, which were derived from the $125-\mathrm{kb}$ plasmid through deletions (Appendix Figure, panel C). Accordingly, the composite plasmid was unstable in Salmonella Anatum.

\section{Conclusions}

We identified a new Salmonella Anatum clone that emerged in Taiwan in 2011. During 2011-2014, strains of the new clone were not resistant and caused few infections. The dramatic increase in Salmonella Anatum infections that occurred during 2016-2017 was strongly associated with the emergence of MDR strains in 2015. The most crucial concern regarding emergence of the MDR 
No. SNPs

$18016014012010080 \quad 6040 \quad 20 \quad 0$

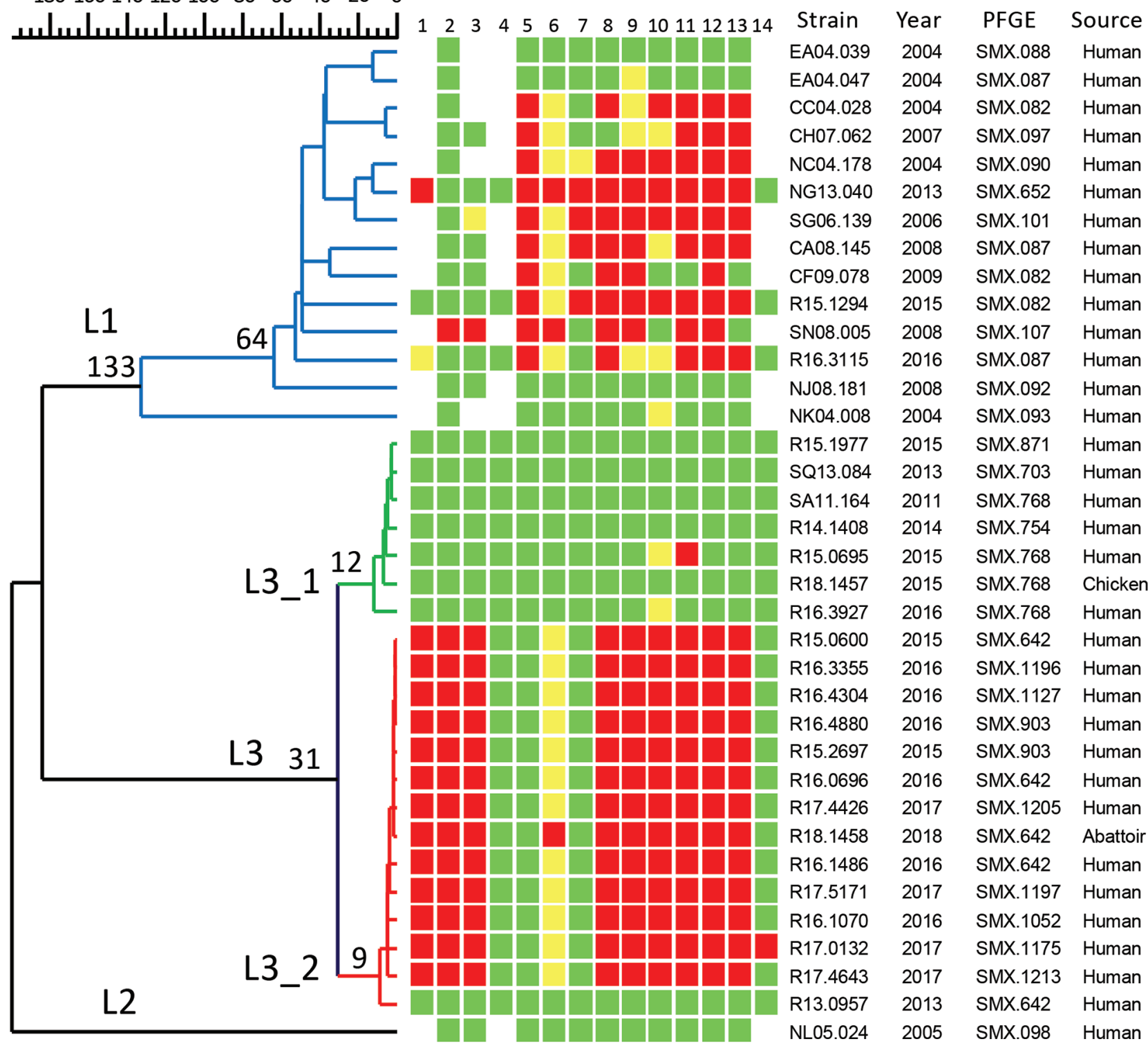

Figure 2. Dendrogram of 36 representative Salmonella enterica serovar Anatum strains from Taiwan, 2004-2017, constructed with wholegenome SNP profiles with 883 SNPs. The complete genomic sequence of Salmonella Anatum strain GT-38 (GenBank accession no. CP013226) was used as the reference for SNP calling. Red, resistant; yellow, intermediate; green, susceptible. Lanes: 1, cefoxitin; 2 , cefotaxime; 3, ceftazidime; 4, ertapenem; 5, nalidixic acid; 6, ciprofloxacin; 7, gentamicin; 8, ampicillin; 9, chloramphenicol; 10, streptomycin; 11, sulfamethoxazole; 12, tetracycline; 13, sulfamethoxazole/trimethoprim; 14, colistin. L, lineage; PFGE, pulsed-field gel electrophoresis; SNP, single-nucleotide polymorphism. A color version of this figure is available online (http://wwwnc.cdc.gov/EID/article/25/1/18-1103-F2.htm).

Salmonella Anatum clone was that all MDR strains carry $b l a_{\mathrm{DHA}-1}$, which encodes AmpC $\beta$-lactamase and confers resistance to $\beta$-lactam drugs, including third-generation cephalosporins. This resistance cannot be overcome by using $\beta$-lactam inhibitors. Because these MDR strains can cause numerous infections in humans and are prevalent in animals used for food, urgent control measures are needed.

\section{Acknowledgments}

We thank the Bureau of Animal and Plant Health Inspection and Quarantine, Council of Agriculture and Agricultural Technology Research Institute, for providing Salmonella Anatum isolates recovered from chicken carcasses and abattoir environments.

This study was funded by the Ministry of Health and Welfare, Taiwan (grant no. MOHW107-CDC-C-315-124503). 


\section{About the Author}

Dr. Chiou is a principal investigator at the Centers for Disease Control, Ministry of Health and Welfare, Taiwan. His research interests include genotyping, molecular epidemiology, and antimicrobial resistance of foodborne bacterial pathogens.

\section{References}

1. Guibourdenche M, Roggentin P, Mikoleit M, Fields PI, Bockemühl J, Grimont PA, et al. Supplement 2003-2007 (no. 47) to the White-Kauffmann-Le Minor scheme. Res Microbiol. 2010;161:26-9. http://dx.doi.org/10.1016/ j.resmic.2009.10.002

2. Kuo HC, Lauderdale TL, Lo DY, Chen CL, Chen PC, Liang SY, et al. An association of genotypes and antimicrobial resistance patterns among Salmonella isolates from pigs and humans in Taiwan. PLoS One. 2014;9:e95772. http://dx.doi.org/10.1371/ journal.pone.0095772

3. Ribot EM, Fair MA, Gautom R, Cameron DN, Hunter SB, Swaminathan B, et al. Standardization of pulsed-field gel electrophoresis protocols for the subtyping of Escherichia coli O157:H7, Salmonella, and Shigella for PulseNet. Foodborne Pathog Dis. 2006;3:59-67. http://dx.doi.org/10.1089/fpd.2006.3.59

4. Chiou CS, Torpdahl M, Liao YS, Liao CH, Tsao CS, Liang SY, et al. Usefulness of pulsed-field gel electrophoresis profiles for the determination of Salmonella serovars. Int J Food Microbiol. 2015;214:1-3. http://dx.doi.org/10.1016/j.ijfoodmicro.2015.07.016

5. Fernández-Alarcón C, Singer RS, Johnson TJ. Comparative genomics of multidrug resistance-encoding IncA/C plasmids from commensal and pathogenic Escherichia coli from multiple animal sources. PLoS One. 2011;6:e23415. http://dx.doi.org/10.1371/ journal.pone. 0023415

6. Cabezón E, Ripoll-Rozada J, Peña A, de la Cruz F, Arechaga I. Towards an integrated model of bacterial conjugation. FEMS Microbiol Rev. 2015;39:81-95.

Address for correspondence: Chien-Shun Chiou, Centers for Disease Control, Center for Diagnostics and Vaccine Development, 5F No. 20, Wen-Sin South Third Rd, Taichung 40855, Taiwan; email: nipmcsc@cdc.gov.tw

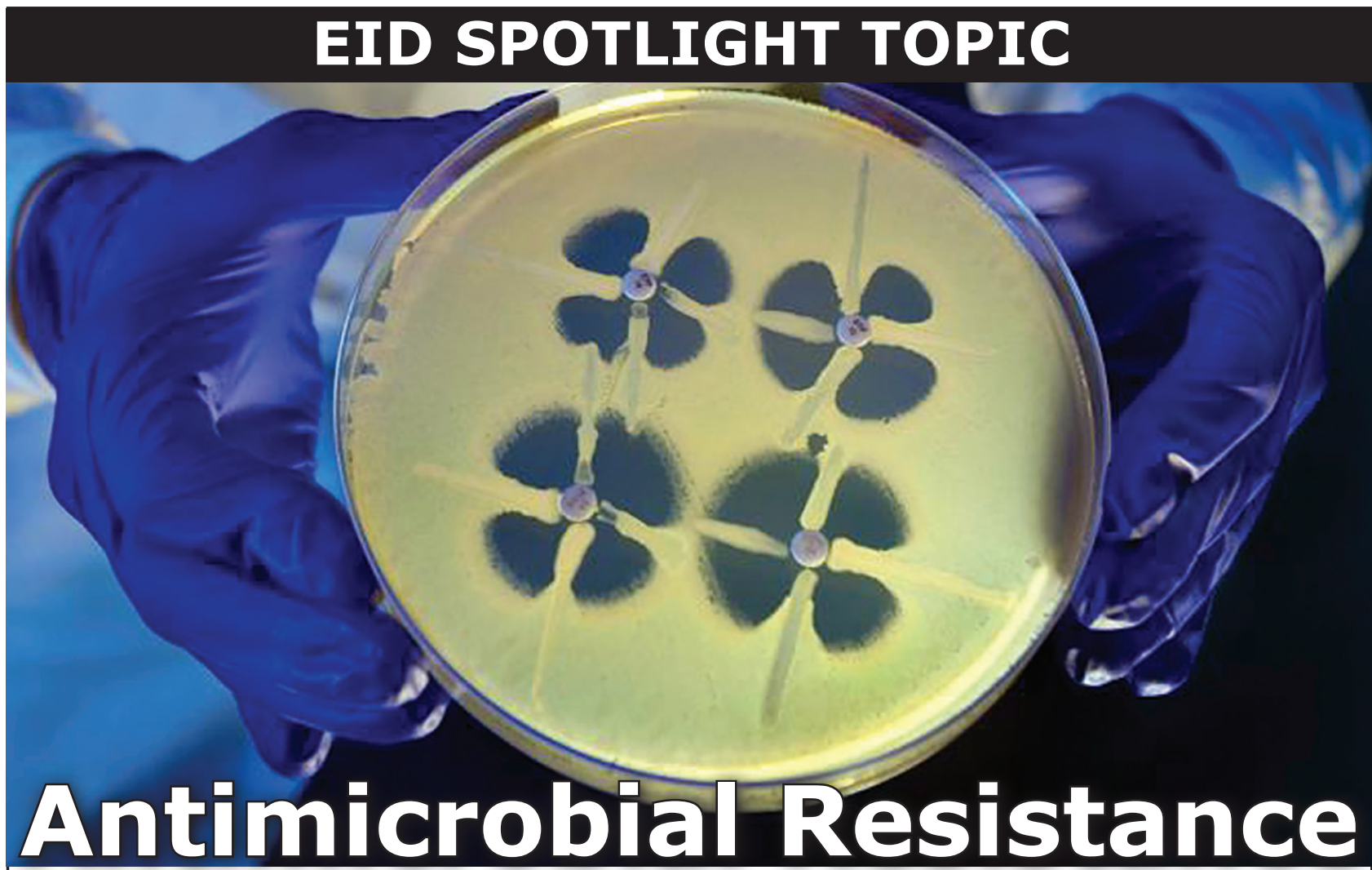

Antibiotics and similar drugs, together called antimicrobial agents, have been used for the past 70 years to treat patients who have infectious diseases. Since the 1940s, these drugs have greatly reduced illness and death from infectious diseases. However, these drugs have been used so widely and for so long that the infectious organisms the antibiotics are designed to kill have adapted to them, making the drugs less effective.

Each year in the United States, at least 2 million people become infected with bacteria that are resistant to antibiotics and at least 23,000 people die each year as a direct result of these infections.

\section{EMERGING}

INFECTIOUS DISEASES http://wwwnc.cdc.gov/eid/page/resistance-spotlight 